\title{
A model for the condensation of a dusty plasma
}

\author{
P. M. Bellan \\ Applied Physics, California Institute of Technology, Pasadena, California 91125
}

(Received 5 January 2004; accepted 23 March 2004; published online 27 May 2004)

\begin{abstract}
A model for the condensation of a dusty plasma is constructed by considering the spherical shielding layers surrounding a dust grain test particle. The collisionless region less than a collision mean free path from the test particle is shown to separate into three concentric layers, each having distinct physics. The method of matched asymptotic expansions is invoked at the interfaces between these layers and provides equations which determine the radii of the interfaces. Despite being much smaller than the Wigner-Seitz radius, the dust Debye length is found to be physically significant because it gives the scale length of a precipitous cut-off of the shielded electrostatic potential at the interface between the second and third layers. Condensation is predicted to occur when the ratio of this cut-off radius to the Wigner-Seitz radius exceeds unity and this prediction is shown to be in good agreement with experiments. (C) 2004 American Institute of Physics.
\end{abstract}

[DOI: $10.1063 / 1.1740773$ ]

\section{INTRODUCTION}

Condensation of a dusty plasma ${ }^{1}$ into a crystalline state was proposed by Ikezi in $1986^{2}$ and demonstrated experimentally eight years later by a number of research groups. ${ }^{3-6}$ The subject has been reviewed by Morfill et al. ${ }^{7}$ and most recently an experiment to test dusty plasma physics has been set up on the International Space Station. ${ }^{8}$

The original model ${ }^{2}$ for this process was motivated by Monte Carlo calculations ${ }^{9}$ which predicted that a Coulomb crystal would form when the Coulomb interaction energy between two adjacent charged particles in a one-componentplasma exceeded their thermal energy by some factor. The Coulomb interaction energy for charged particles with density $n$ is the electrostatic energy of one particle in the potential of an adjacent particle located at the Wigner-Seitz interparticle separation distance ${ }^{10}$

$$
a=\left(\frac{3}{4 \pi n}\right)^{1 / 3} .
$$

According to the Monte Carlo calculations, condensation of charged particles into a crystal should occur when

$$
\Gamma=\frac{Z^{2} e^{2}}{4 \pi \epsilon_{0} a \kappa T} \gtrsim 170,
$$

where $Z$ is the charge on each particle and $T$ is the temperature of the particles. As noted by Ikezi, ${ }^{2}$ Eq. (2) could be a very poor estimate for dusty plasmas (which are a threecomponent-plasma), but lacking a better model, Eq. (2) has often been used as a benchmark for dusty plasma crystallization experiments. The experiments show ${ }^{6}$ that the actual value of $\Gamma$ required for condensation is two to three orders of magnitude larger than that predicted by Eq. (2). Thus, while Ikezi's original postulate that dusty plasmas can condense into crystals has been experimentally validated, there has not been a quantitative model predicting the value of $\Gamma$ necessary for condensation to occur.
Interactions between adjacent particles in a plasma are intimately related to the concept of Debye shielding. According to this concept, any plasma particle can be considered to be a test particle surrounded by a screening cloud of adjacent particles. The screening completely cancels the test particle field at distances much greater than the Debye length. Screening may be accomplished by adjacent particles of either the same polarity as the test charge or opposite polarity, but is subject to the constraint that the test particle cannot be moving faster than the thermal velocity of the shielding particles. ${ }^{11}$ For example, if the test particle is an electron, it is shielded by the repulsion of other electrons in the presence of a uniform neutralizing ion background, but it is not shielded by ions because it is moving too fast for ions to respond. On the other hand, ions are shielded by both electrons and ions. In a dusty plasma one might thus reasonably expect dust grains to be shielded by electrons, ions, and other dust grains.

The standard model of Debye shielding is based on the Boltzmann relation, an equilibrium solution to the fluid equation of motion for each species $\sigma$ such that the force due to the electric field balances the force due to the gradient of an isotropic scalar pressure, i.e.,

$$
0=-n_{\sigma} q_{\sigma} \boldsymbol{\nabla} \phi-\nabla P_{\sigma} .
$$

Three critical assumptions are intrinsic to the standard model of Debye shielding, namely: (i) it is assumed that the plasma is sufficiently collisional that the concept of an isotropic scalar pressure $P_{\sigma}=n_{\sigma} \kappa T_{\sigma}$ is valid, (ii) it is assumed that a Boltzmann dependence $n_{\sigma}=n_{\sigma 0} \exp \left(-q_{\sigma} \phi / \kappa T_{\sigma}\right)$ exists relating the local density $n_{\sigma}$ to the system-averaged density $n_{\sigma 0}$, and (iii) it is assumed that $\left|q_{\sigma} \phi / \kappa T_{\sigma}\right| \ll 1$ so that the Boltzmann relationship may be linearized giving $n_{\sigma} / n_{\sigma 0}=1$ $-q_{\sigma} \phi / \kappa T_{\sigma}$. The standard model for Debye shielding of a test particle with charge $q_{T}$ results when the linearized Boltzmann relationships of the various species are substituted into Poisson's equation giving the Yukawa-type solution $\phi(r)=q_{T} \exp \left(-r / \lambda_{\mathrm{D}}\right) / 4 \pi \epsilon_{0} r$ where 


$$
\frac{1}{\lambda_{\mathrm{D}}^{2}}=\sum \frac{1}{\lambda_{\mathrm{D} \sigma}^{2}}
$$

and

$$
\lambda_{\mathrm{D} \sigma}^{2}=\frac{\epsilon_{0} \kappa T_{\sigma}}{n_{\sigma 0} q_{\sigma}^{2}} .
$$

The summation in Eq. (4) is restricted to species that participate in the shielding and so excludes all species having thermal velocity slower than the species of the test particle.

When $r \rightarrow 0$, the Yukawa solution diverges, violating assumption (iii) that $\left|q_{\sigma} \phi / \kappa T_{\sigma}\right| \ll 1$ and causing the standard model to fail to be internally self-consistent. This failure of the standard model of Debye shielding has been noted previously, see, e.g., Lampe, Joyce, and Ganguli ${ }^{12}$ for criticism regarding assumptions (i)-(iii). In addition, Hansen and Fajans ${ }^{13}$ have shown that trapping can affect Debye shielding in a pure electron plasma while Goree, ${ }^{14}$ Zobnin et al.,${ }^{15}$ and Lampe et al. ${ }^{16}$ have shown that trapping of ions can affect shielding of a dust grain.

The issue of how to treat Debye shielding when $\left|q_{\sigma} \phi / \kappa T_{\sigma}\right| \gg 1$ is especially critical for the dust condensation problem, because $\left|q_{\sigma} \phi / \kappa T_{\sigma}\right|$ is essentially the same as $\Gamma$. Consensus does not exist on how to address this issue.

Furthermore, the form of Eq. (4) is such that the sum on the right-hand side is dominated by the term having the smallest $\lambda_{\mathrm{D} \sigma}^{2}$ and, since dust particles are both cold and highly charged, the dust Debye length is typically much smaller than both the electron and ion Debye lengths. One might expect that the system Debye length $\lambda_{\mathrm{D}}$ should be very nearly the dust Debye length $\lambda_{\mathrm{D} d}$, but this point of view has usually been rejected. The dust Debye length $\lambda_{\mathrm{D} d}$ is typically so small that it is less than $a$, and questions have been raised as to whether such a short shielding length has physical significance since the standard Debye argument is based on the implicit assumption that there is a statistically large number of particles in a sphere having the Debye radius. This is clearly not true for dust particles in a sphere with radius $\lambda_{\mathrm{D} d}$ if $\lambda_{\mathrm{D} d}$ is less than $a$. Nevertheless, Wang and Bhattarcharjee ${ }^{17}$ and also Otani and Bhattarcharjee ${ }^{18}$ argued that some sort of shielding does occur at the scale of $\lambda_{\mathrm{D} d}$ but the only support for this point of view was the demonstration ${ }^{18}$ of some time-averaged correlation effects at the scale of $\lambda_{\mathrm{D} d}$ in a one-dimensional numerical simulation that would only crystallize if artificially annealed. Most other authors ignore dust self-shielding on the presumption that the Debye shielding concept does not make sense when a Debye length is smaller than $a$.

We present here a model for a dusty plasma on the verge of condensation. This model takes into account both collisional and collisionless behavior in three-dimensional geometry, avoids inappropriate use of fluid theory, shows that the dust Debye length has important physical significance even though it is much smaller than $a$, and predicts a condensation threshold in good agreement with experimental measurements. The derivation identifies four physically distinct concentric regions surrounding a test charge. The ions are collisionless in the innermost three regions but collisional in the outer, fourth region (the collisionless nature of ions in the inner three regions is consistent with the assumptions inherent in dust grain charging theory). The method of matched asymptotic expansions is used to locate the two interfaces between the first three regions and knowledge of these interface locations is then used to give the criterion for condensation. The $T_{e} \gg T_{i}, T_{d}$ temperature regime of typical dusty plasma condensation experiments is assumed and ions are assumed to be singly charged (the theory could be extended to arbitrary temperatures without great difficulty, but this would unnecessarily complicate the model).

The paper is organized as follows: Section II reviews relevant aspects of dust charging theory and sets up a dimensionless parameter space suitable for comparing the model to experiments. Section III uses collisionless Vlasov theory to calculate the ion, electron, and dust grain densities and shows that when $\left|e \phi / \kappa T_{i}\right|>1$, the ion density differs from the Boltzmann model; this difference demonstrates the inappropriateness of fluid models in this regime and resolves the paradox associated with divergence of the Yukawa solution at small $r$. Section IV shows that the vicinity of a test particle can be divided into three concentric spherical regions each having distinct physics determined by the magnitude of $\left|e \phi / \kappa T_{i}\right|$. Section V derives approximate solutions to the Vlasov-Poisson system for these three regions and Sec. VI derives matching conditions across the two interfaces between the three regions. Section VII uses the matching conditions to deduce a condition for dust condensation and compares the model predictions with experiments. Section VIII provides a summary and discussion.

\section{DUST CHARGING AND DUSTY PLASMA PARAMETER SPACE}

Two independent parameters characterize the dust grains in a dusty plasma: the grain radius $r_{d}$ and the Wigner-Seitz radius $a$. In order to develop a model based on dimensionless parameters, the ion Debye length

$$
\lambda_{\mathrm{D} i}=\sqrt{\frac{\epsilon_{0} \kappa T_{i}}{n_{i 0} e^{2}}}
$$

will be used as the "yardstick" by which all lengths are measured. A bar will be used to denote lengths normalized to the ion Debye length so that the normalized Wigner-Seitz radius, for example, is

$$
\bar{a}=\frac{1}{\lambda_{\mathrm{D} i}}\left(\frac{3}{4 \pi n_{d 0}}\right)^{1 / 3} .
$$

The two quantities $\bar{a}$ and $\bar{r}_{d}$ constitute the coordinates for a dimensionless dusty plasma parameter space.

In order to avoid confusing minus signs, the electrostatic potential $\phi$ will be replaced by the positive dimensionless variable

$$
\psi=-\frac{e \phi}{\kappa T_{i}}
$$


and $\psi_{d}$ will denote the potential on the surface of a dust grain. Thus, positive $\psi$ attracts ions but repels both electrons and dust grains.

When dust grains are placed in an electron-ion plasma, some fraction of the electrons attach to the dust grain surface, causing the dust grains to become negatively charged and reducing the density of free electrons. The quantitative theory of dust charging, summarized in Ref. 1, combines collisionless Vlasov theory with an analysis of trajectories of individual particles as they approach a finite radius charged sphere. The particle trajectories are assumed to be governed by orbital-motion-limited (OML) theory ${ }^{19-21}$ wherein particle trajectories are assumed to be collisionless and completely determined by considerations of conservation of angular momentum and conservation of energy. There has been some question ${ }^{6}$ about the extent to which the standard dust charging model applies to dust grains in an electrode sheath, the typical situation for terrestrial dusty plasma condensation experiments, but not for the zero-gravity dusty plasma condensation experiment on the International Space Station. We assume in this paper that the standard dust charging model is applicable so that the effect, if any, of electrode sheaths on dust charging is small.

The standard dust charging model shows that dust grain charging is governed by the dimensionless parameter

$$
P=4 \pi n_{d 0} \lambda_{\mathrm{D} i}^{2} r_{d}=4 \pi n_{d 0} \lambda_{\mathrm{D} i}^{3} \bar{r}_{d}=\frac{3 \bar{r}_{d}}{\bar{a}^{3}}
$$

where $P$ has the functional dependence

$$
P=\frac{1}{\psi_{d}}-\left(1+\frac{1}{\psi_{d}}\right) \sqrt{\frac{m_{e} T_{i}}{m_{i} T_{e}}} \exp \left(\psi_{d} T_{i} / T_{e}\right) .
$$

Global quasineutrality gives

$$
Z_{d} n_{d 0}+n_{e 0}=n_{i 0},
$$

where $Z_{d}$ is the number of electrons captured by a dust grain. We define the electron capture factor

$$
\alpha=Z_{d} n_{d 0} / n_{i 0}
$$

so that $\alpha=1$ corresponds to having all the electrons attached to the dust grains while $\alpha=0$ corresponds to having no electrons attached to the dust grains. The quasineutrality condition, Eq. (11), can thus be expressed as

$$
\alpha+\frac{n_{e 0}}{n_{i 0}}=1
$$

and dust charging theory ${ }^{22}$ shows that

$$
\alpha=P \psi_{d} .
$$

Since $\psi_{d}$ and $\alpha$ are functions of $P$, they have functional dependence $\alpha=\alpha\left(\bar{a}, \bar{r}_{d}\right)$ and $\psi_{d}=\psi_{d}\left(\bar{a}, \bar{r}_{d}\right)$.

Combining Eqs. (9), (12), and (14) shows that

$$
\frac{Z_{d}}{4 \pi n_{i 0} \lambda_{\mathrm{D} i}^{3}}=\bar{r}_{d} \psi_{d},
$$

so that $Z_{d}$ becomes large if $\psi_{d}$ is finite, $\bar{r}_{d}$ is not infinitesimal, and $4 \pi n_{i 0} \lambda_{\mathrm{D} i}^{3}$ is large. Equation (15) is just the normal- ized version of the potential $\phi_{d}=-Z_{d} e / 4 \pi \epsilon_{0} r_{d}$ of a sphere of radius $r_{d}$ with surface charge $-Z_{d} e$. This result is actually slightly incorrect for a shielded dust grain, because, as shown in the next paragraph, the shielding cloud surrounding a dust grain depresses the potential at the grain surface to a value below the value given by Eq. (15).

To understand this potential depression effect due to shielding, consider the potential $\phi$ on the surface of a sphere with charge $Q$ and radius $r_{\text {sphere }}$ surrounded by a shell of shielding charge $-Q$ at radius $r_{\text {shell }}$. The potential on the surface of the shielded sphere is given by

$$
\phi\left(r_{\text {sphere }}\right)=\int_{\infty}^{r_{\text {sphere }}} d r \frac{\partial \phi}{\partial r}=\frac{Q}{4 \pi \epsilon_{0}}\left(\frac{1}{r_{\text {sphere }}}-\frac{1}{r_{\text {shell }}}\right),
$$

a result obtained by taking into account the contributions to $\partial \phi / \partial r$ from both the sphere and its shielding charge. The ratio of the surface potential of the shielded sphere to the potential of an identical unshielded sphere is $\phi\left(r_{\text {sphere }}\right) / \phi_{\text {vac }}=1-r_{\text {sphere }} / r_{\text {shell }}$, where $\phi_{\text {vac }}$ is the surface potential of the unshielded sphere. If $r_{\text {shell }}-r_{\text {sphere }} \simeq \lambda_{\mathrm{D}}$ where $\lambda_{\mathrm{D}}$ is the nominal Debye length, then $\phi\left(r_{\text {sphere }}\right) / \phi_{\text {vac }}$ $\simeq \lambda_{\mathrm{D}} /\left(r_{\text {sphere }}+\lambda_{\mathrm{D}}\right)$ so the potential of the shielded sphere will be greatly depressed from its vacuum value if $r_{\text {sphere }}$ $\gg \lambda_{\mathrm{D}}$. This indicates that being highly charged is insufficient for a dust grain to have a large potential; it also needs to have $\bar{r}_{d} \ll 1$. The model of dust charging thus has the implicit assumption that $\bar{r}_{d}$ is small compared to unity and this assumption will be made in the remainder of this paper.

Figures 1(a)-1(c) show contours of constant $\psi_{d}, \alpha$, and $Z_{d} / 4 \pi n_{i 0} \lambda_{\mathrm{D} i}^{3}$ as determined by Eqs. (9), (10), (14), and (15) for the parameters of Ref. 3, a typical dust crystallization experiment. Since dust grains in a given experiment have a fixed ratio $\bar{r}_{d} / \bar{a}$, a specific experiment is characterized by a sloping straight line in $\bar{a}, \bar{r}_{d}$ parameter space. Moving up and to the right along such a line corresponds to making $\lambda_{d i}$ smaller whereas moving down and to the left corresponds to making $\lambda_{d i}$ larger. Densities in an experiment are typically measured by Langmuir probes which have an uncertainty of $-50 \%,+100 \%$, so that the density of $n_{i}=10^{9} \mathrm{~cm}^{-3}$ reported in Ref. 3 would actually be in the range $5 \times 10^{8} \mathrm{~cm}^{-3}<n_{i}$ $<2 \times 10^{9} \mathrm{~cm}^{-3}$. This factor of 4 range of densities ${ }^{4}$ corresponds to the straight line segment labeled "expt" in Figs. 1(a)-1(c). This line has a slope given by $\bar{r}_{d} / \bar{a}=r_{d} / a$. The left end of this line is the point in parameter space calculated using the lower estimate for the density, while the right end corresponds to using the upper estimate for the density. The length of this line effectively represents the density measurement error bar. To the extent that charging theory is correct, the range of possible values of $\alpha, \psi_{d}$, and $Z_{d} / 4 \pi n_{i 0} \lambda_{\mathrm{D} i}^{3}$ for the experiment is given by the intersection of the contours with this "expt" line.

\section{VLASOV MODEL OF CHARGED PARTICLE DENSITY IN THE PRESENCE OF A POTENTIAL}

A typical dust grain will be considered as a test particle inserted in a plasma consisting of electrons, ions, and other dust grains. The origin of a spherical coordinate system will be defined to be at the center of this test particle. Typical dust 

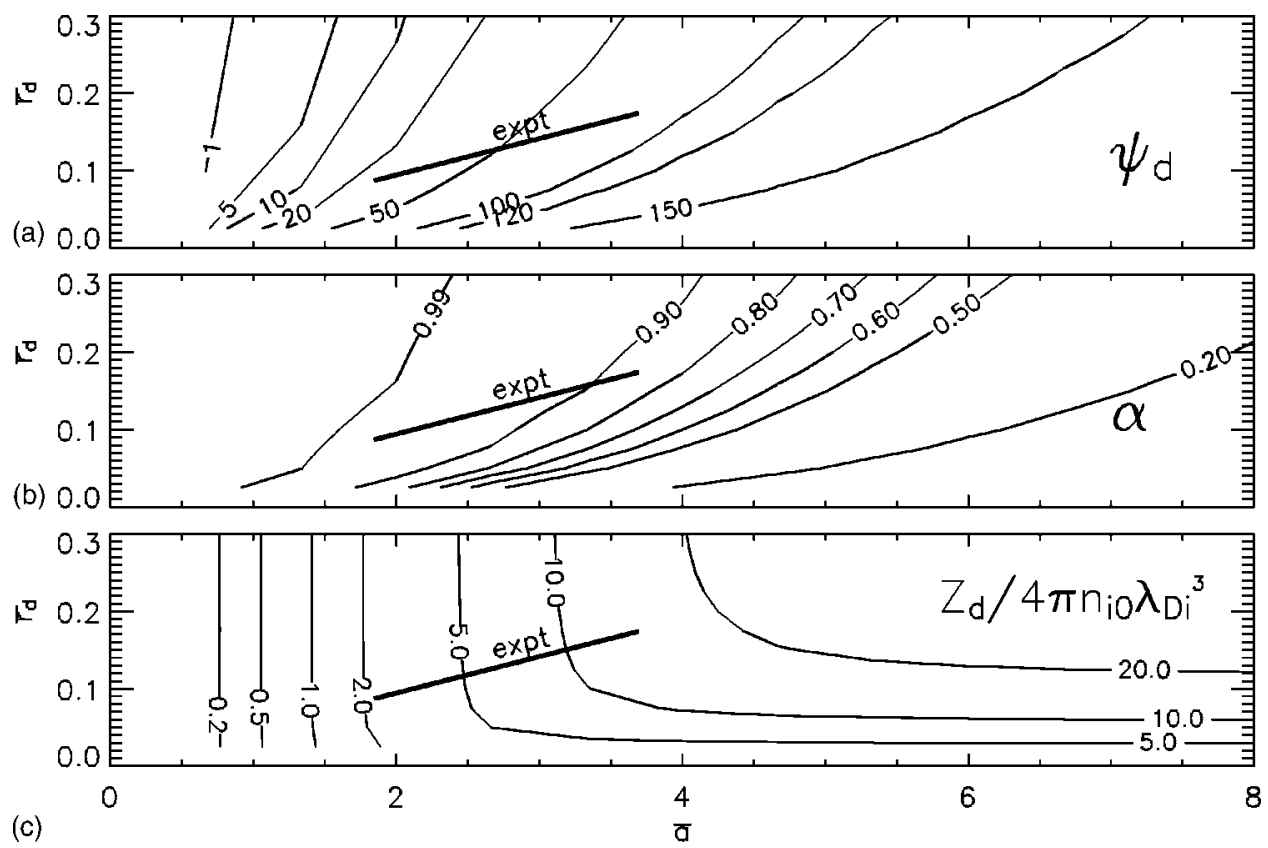

FIG. 1. Dusty plasma parameter space for Chu and I experiment (density range indicated by short line labeled "expt"): horizontal axis is $\bar{a}=a / \lambda_{d i}$, vertical axis is $\bar{r}_{d}=r_{d} / \lambda_{d i}$; (a) contours of constant $\psi_{d}$, (b) contours of constant $\alpha$, (c) contours of constant $Z_{d} / 4 \pi n_{i 0} \lambda_{d i}^{3}$.

condensation experiments have neutral pressures $\sim 10^{2} \mathrm{~Pa}$ (corresponding to a neutral density $n_{n} \simeq 3 \times 10^{16} \mathrm{~cm}^{-3}$ ). Since neutral cross sections are $\sigma \sim 3 \times 10^{-16} \mathrm{~cm}^{2}$, the mean free path for ion-neutral collisions is $l_{\mathrm{mfp}}=\left(n_{n} \sigma\right)^{-1}$ $\sim 1 \mathrm{~mm}$, which is at least an order of magnitude larger than shielding scale lengths. The last collision experienced by an ion in the vicinity of the test particle will have occurred outside a sphere having a normalized diameter of the order of $\bar{l}_{\text {mfp }}$; such a sphere is shown schematically in Fig. 2 and lies at the interface between regions 3 and 4 . Thus ions can be

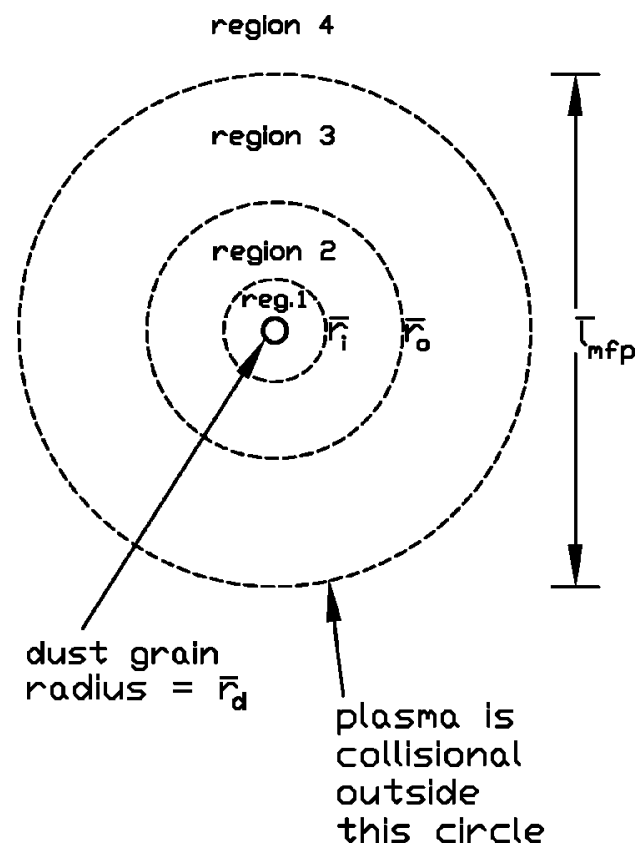

FIG. 2. Sketch of concentric regions surrounding a dust grain test particle of normalized radius $\bar{r}_{d}$. Diameter of outermost dashed circle is $\bar{l}_{\mathrm{mfp}}$, the normalized mean free path for collisions, so plasma is collisional in region 4 outside this circle. Regions 1, 2, and 3 are collisionless and have interfaces on the dashed circles having normalized radii $\bar{r}_{i}$ and $\bar{r}_{o}$. considered collisionless inside regions $1-3$ and collisional in region 4; the details of regions $1-3$ will be discussed later. This separation of space into collisional and collisionless regions is similar to the arguments used in dust grain charging theory (the OML assumption underlying dust grain charging theory is based on angular momentum conservation which can only be true if a particle has no collisions).

Electrostatic potential is undefined with respect to a constant; following convention, we choose this constant such that $\phi=0$ at infinity. Collisions make the distribution function Maxwellian in region 4 and this provides a boundary condition for the collisionless distribution function in regions $1-3$. The distribution function in regions 1-3 must satisfy the collisionless Vlasov equation and so must be must be a function of constants of the motion. ${ }^{23,24}$ The relevant constant of the motion here is the particle energy $W=m_{\sigma} v^{2} / 2$ $+q_{\sigma} \phi$, and so the distribution function in regions $1-3$ is

$$
f_{\sigma}(\mathbf{r}, \mathbf{v})=n_{\sigma 0}\left(\frac{m_{\sigma}}{2 \pi \kappa T_{\sigma}}\right)^{3 / 2} \exp \left(-\frac{m_{\sigma} v^{2} / 2+q_{\sigma} \phi(\mathbf{r})}{\kappa T_{\sigma}}\right) .
$$

This is the right choice because $f_{\sigma}(\mathbf{r}, \mathbf{v})$ is not only a function of a constant of the motion $W$ but also joins smoothly to the region 4 Maxwellian solution where $\phi=0$ and the plasma is collisional.

Electrons and dust grains experience a repulsive force upon approaching the negatively charged dust grain test particle and so are slowed down with some particles being slowed down to zero velocity and reflecting. Thus, electrons or dust grains near the dust grain test particle can have zero velocity. The respective electron and dust grain densities in the vicinity of the dust grain test particle are thus given by

$$
n_{e}=\int_{0}^{\infty} 4 \pi v^{2} d v f_{e}(\mathbf{r}, \mathbf{v})=n_{e 0} \exp \left(\frac{e \phi(\mathbf{r})}{\kappa T_{e}}\right)
$$




$$
n_{d}=\int_{0}^{\infty} 4 \pi v^{2} d v f_{d}(\mathbf{r}, \mathbf{v})=n_{d 0} \exp \left(\frac{Z_{d} e \phi(\mathbf{r})}{\kappa T_{d}}\right) .
$$

Using Eq. (8), the electron and dust grain densities normalized to their average values are

$$
\frac{n_{e}}{n_{e 0}}=\exp \left(-\psi T_{i} / T_{e}\right)
$$

and

$$
\frac{n_{d}}{n_{d 0}}=\exp \left(-Z_{d} \psi T_{i} / T_{d}\right) .
$$

These densities are identical to the Boltzmann relation and so demonstrate that collisionless kinetic theory agrees with fluid theory for negatively charged particles near a negatively charged test particle.

Ion behavior is fundamentally different because ions, being positive, are accelerated as they approach the negatively charged dust grain test particle; this means that there are no zero velocity ions near a dust grain. The slowest ion is one that has fallen into the negative $\phi$ well with zero initial velocity at the edge of the well and the velocity of such an ion will satisfy

$$
m_{i} v^{2} / 2+e \phi=0 .
$$

Using Eq. (8) it is seen that this minimum possible ion velocity can be expressed as

$$
v_{\min }=\sqrt{\frac{2 \kappa T_{i}}{m_{i}} \psi} .
$$

Evaluation of the ion density in the vicinity of the dust grain test particle therefore requires invoking a lower limit at $v_{\min }$ for the velocity integration over the distribution function. The resulting ion density is thus

$$
\begin{aligned}
n_{i}= & \int_{v_{\min }}^{\infty} 4 \pi v^{2} d v f_{i}(\mathbf{r}, \mathbf{v}) \\
= & n_{i 0}\left(\frac{m_{i}}{2 \pi \kappa T_{i}}\right)^{3 / 2} \exp (\psi) \int_{\sqrt{2 \kappa T_{i} \psi / m_{i}}}^{\infty} \\
& \times 4 \pi v^{2} d v \exp \left(-\frac{m_{i} v^{2}}{2 \kappa T_{i}}\right) .
\end{aligned}
$$

By defining $\xi=v / \sqrt{2 \kappa T_{i} / m_{i}}$, the normalized ion density can be expressed as

$$
\begin{aligned}
\frac{n_{i}}{n_{i 0}} & =\frac{4 e^{\psi}}{\sqrt{\pi}} \int_{\sqrt{\psi}}^{\infty} \xi^{2} d \xi \exp \left(-\xi^{2}\right) \\
& =e^{\psi}(1-\operatorname{erf} \sqrt{\psi})+\frac{2}{\sqrt{\pi}} \sqrt{\psi},
\end{aligned}
$$

where

$$
\operatorname{erf} z=\frac{2}{\sqrt{\pi}} \int_{0}^{z} e^{-\xi^{2}} d \xi
$$

is the error function. The second line in Eq. (25) is obtained using the identity

$$
\begin{aligned}
\int_{\sqrt{\psi}}^{\infty} d \xi \frac{d}{d \xi}\left(\xi \exp \left(-\xi^{2}\right)\right)= & \int_{\sqrt{\psi}}^{\infty} d \xi \exp \left(-\xi^{2}\right) \\
& -2 \int_{\sqrt{\psi}}^{\infty} d \xi \xi^{2} \exp \left(-\xi^{2}\right) .
\end{aligned}
$$

For small arguments, the error function may be approximated,

$$
\lim _{z \rightarrow 0} \operatorname{erf} z=\frac{2}{\sqrt{\pi}}\left(z-\frac{z^{3}}{3}\right) .
$$

Thus for $\psi \ll 1$ and hence $\sqrt{\psi} \ll 1$, the normalized ion density has the form

$$
\begin{aligned}
\frac{n_{i}}{n_{i 0}} & =\left(1+\psi+\frac{1}{2} \psi^{2}\right)\left(1-\frac{2}{\sqrt{\pi}}\left(\sqrt{\psi}-\frac{\psi^{3 / 2}}{3}\right)\right)+\frac{2}{\sqrt{\pi}} \sqrt{\psi} \\
& \simeq 1+\psi,
\end{aligned}
$$

which is the same as the Boltzmann result given by fluid theory.

However, because

$$
\lim _{\psi \rightarrow \infty} e^{\psi}[1-\operatorname{erf}(\sqrt{\psi})]=0,
$$

the normalized ion density when $\psi \gg 1$ is

$$
\frac{n_{i}}{n_{i 0}} \simeq \frac{2}{\sqrt{\pi}} \sqrt{\psi}
$$

this is much smaller than the fluid theory Boltzmann relation prediction that $n_{i} / n_{i 0}=\exp (\psi)$. Equation (30) thus demonstrates a failure of fluid theory and its associated Boltzmann relationship when $\psi \gg 1$. This failure occurs because the concept of ion pressure no longer makes sense when $\psi \gg 1$. The pressure concept is based on the assumption that particles have an isotropic Gaussian distribution of random velocities about some mean velocity whereas when $\psi \gg 1$, ions in reality are falling into a deep potential well and do not have a random distribution of velocities about some mean velocity. Equation (25) and the distinction between its small and large $\psi$ limits have been previously discussed by Laframboise and Parker ${ }^{25}$ in the context of electrostatic probes and by Lampe, Joyce, and Ganguli ${ }^{12}$ in the context of dusty plasmas.

The lower limit of the integral in Eq. (24) causes the ion distribution to have an $r-v$ phase-space "hole" in the vicinity of the dust grain since $f(r, v)=0$ for velocities below $v_{\min }$. It has been argued by Bernstein and Rabinowitz, ${ }^{26}$ Laframboise and Parker, ${ }^{25}$ and Lampe ${ }^{27}$ that for a certain class of radial potential profiles, another sort of phase-space hole can also exist. This additional hole results from a rather subtle barrier that can occur because for a certain range of the angular momentum $J$ the effective potential $U_{\text {eff }}(r)$ $=q \phi(r)+J^{2} / 2 m r^{2}$ can have a small local maximum. This barrier prevents access to small $r$ by particles having a cer- 
tain range of $W$ and $J$. If such a barrier exists, the radial ion density profile will differ somewhat from the predictions of OML theory, because the ions that cannot pass by this barrier will have a radial turning point at a larger radius than predicted by OML. However, since Poisson's equation shows that $\psi$ is essentially a double integral of the net charge density up to a radius $r$, changes in the turning point of small classes of ions should not have a major effect on the $\psi$ profile, i.e., small corrections to the OML model should not result in a significant collective effect. Lampe ${ }^{27}$ has shown that the error introduced by omission of consideration of these centrifugal force barriers is very small for dusty plasmas and so we will ignore this correction to OML theory.

Another correction to OML theory results from consideration of ion capture by the dust grain which also causes a hole in phase-space. ${ }^{20,26}$ As shown in Ref. 26 capture of ions by the dust grain reduces the number of ions moving radially outward from the dust grain in comparison to the limiting situation where the dust grain does not capture any ions so that all ions are perfectly reflected from the dust grain. Taking into account the reduction in the number of outward moving ions compared to inward moving ions would require replacing the distribution function prescribed by Eq. (17) by a distribution function of the form ${ }^{26} f=f_{+}+f_{-}$, where $f_{+}(W)$ is the phase space density of ions moving radially outwards from the dust grain and $f_{-}(W)$ is the phase space density of ions moving radially inwards. If ions are perfectly reflected at the dust grain then $f_{+}=f_{-}$in which case Eq. (17) is appropriate, but if ions are captured by the dust grain then $f_{+}<f_{-}$and a more complicated prescription than Eq. (17) would have to be used. We will assume that the fraction of ions incident at $\bar{r}_{o}$ which are captured by the dust grain is so small that Eq. (17) is a reasonably accurate prescription for the ion phase space density. We are thus assuming that the $r$ projection of ion motion has a reflecting trajectory so that there are equal numbers of ions moving radially inwards and outwards in the dust grain shielding cloud. This assumption will be validated later.

Finally, we will also ignore ion trapping, ${ }^{14-16}$ but will later make some brief comments about the extent to which trapping might be important.

\section{IDENTIFICATION OF THREE REGIONS FOR THE POTENTIAL}

Poisson's equation

$$
\nabla^{2} \phi=-\frac{e}{\epsilon_{0}}\left(n_{i}-n_{e}-Z_{d} n_{d}\right)
$$

relates the densities of the various species to the electrostatic potential. Assuming spherical symmetry about the dust grain test particle and using Eq. (8), Poisson's equation can be recast as

$$
\frac{1}{\bar{r}^{2}} \frac{\partial}{\partial \bar{r}}\left(\bar{r}^{2} \frac{\partial \psi}{\partial \bar{r}}\right)=\frac{n_{i}}{n_{i 0}}-\frac{n_{e 0}}{n_{i 0}} \frac{n_{e}}{n_{e 0}}-Z_{d} \frac{n_{d 0}}{n_{i 0}} \frac{n_{d}}{n_{d 0}}
$$

Using Eqs. (20), (21), and (25) for the normalized densities and also Eq. (12), the normalized Vlasov/Poisson system becomes

$$
\begin{aligned}
\frac{1}{\bar{r}^{2}} \frac{\partial}{\partial \bar{r}}\left(\bar{r}^{2} \frac{\partial \psi}{\partial \bar{r}}\right)= & \underbrace{e^{\psi}(1-\operatorname{erf}(\sqrt{\psi}))+\frac{2}{\sqrt{\pi}} \sqrt{\psi}}_{\text {ions }} \\
& \underbrace{-(1-\alpha) \exp \left(-\frac{\psi T_{i}}{T_{e}}\right)}_{\text {electrons }}-\underbrace{\alpha \exp (-\bar{Z} \psi)}_{\text {dust }},
\end{aligned}
$$

where $\bar{Z}=Z_{d} T_{i} / T_{d}$ is presumed to be large compared to unity since the dust grain is highly charged and $T_{i}>T_{d}$. Equation (33) is a nonlinear ordinary differential equation for $\psi$ and is consistent with the collisionless Vlasov equation. Since the densities were obtained using the collisionless Vlasov equation, this system will be called the Vlasov/ Poisson system to distinguish it from the fluid/Poisson system.

We now argue that three distinct regions exist for $\psi$ such that in each region the Vlasov/Poisson system has a different form. The location of these regions is sketched in Fig. 2 and, going outwards from the surface of the dust grain test particle, these regions and their interfaces are defined by the following:

Region 1 is where $\psi_{d}>\psi>1$ and exists because the grain potential $\psi_{d}$ is large compared to unity [see Fig. 1(a)]. Region 1 is a sheath-like inner region where the ion density has the non-Boltzmann behavior given by Eq. (30).

Region 2 is where $1>\psi>1 / Z_{d}$ and is depleted of dust grains. Shielding in this region is provided mainly by ions.

Region 3 is where $1 / Z_{d}>\psi$ and this region extends to region 4 where collisions set in and where the potential goes to zero. Shielding in region 3 is done mainly by dust grains and this shielding takes place over a very short characteristic length, causing an extremely sharp cut-off of the potential.

The radii of the respective interfaces between regions 1 and 2 and between regions 2 and 3 will be called $\bar{r}_{i}$ and $\bar{r}_{o}$ as indicated in Fig. 2 (the subscripts $i$ and $o$ stand for inner and outer interfaces). The values of $\bar{r}_{i}$ and $\bar{r}_{o}$ will be unknowns to be solved for; determining these radii is the crux of the problem.

\section{APPROXIMATE SOLUTIONS TO THE VLASOV/POISSON SYSTEM FOR THE THREE COLLISIONLESS REGIONS}

The three collisionless regions will now be discussed going from the outermost (region 3) to the innermost (region $1)$. 


\section{A. Region 3 solution: $\psi<1 / \bar{Z}$}

In region 3, Eq. (33) can be approximated as

$$
\begin{aligned}
\frac{1}{\bar{r}^{2}} \frac{\partial}{\partial \partial}\left(\bar{r}^{2} \frac{\partial \psi}{\partial \bar{r}}\right) & =\underbrace{1+\psi}_{\text {ions }}-\underbrace{(1-\alpha)\left(1-\frac{\psi T_{i}}{T_{e}}\right)}_{\text {electrons }}-\underbrace{\alpha(1-\bar{Z} \psi)}_{\text {dust }} \\
& =\left(1+(1-\alpha) \frac{T_{i}}{T_{e}}+\alpha \bar{Z}\right) \psi .
\end{aligned}
$$

Using $T_{e} \gg T_{i}$, this has the Yukawa-type solution

$$
\psi_{3}=\frac{\bar{r}_{o}}{\bar{Z} \bar{r}} \exp \left(-\sqrt{\alpha \bar{Z}+1}\left(\bar{r}-\bar{r}_{o}\right)\right)
$$

The coefficient in Eq. (35) has been chosen so that $\psi_{3}$ $=1 / \bar{Z}$ at $\bar{r}=\bar{r}_{o}$. The effective shielding length in region 3 is the dust Debye length

$$
\lambda_{\mathrm{D} d}=\lambda_{\mathrm{D} i} / \sqrt{1+\alpha \bar{Z}},
$$

which is much smaller than the ion Debye length since $\alpha$ is of order unity [see Fig. 1(b)] and $\bar{Z}=Z_{d} T_{i} / T_{d} \gg 1$.

\section{B. Region 2 solution: $1>\psi>1 / \bar{Z}$}

The $\exp (-\bar{Z} \psi)$ term is dropped from Eq. (33) in region 2 because $\bar{Z} \psi$ is large. Taking into account $T_{i} \ll T_{e}$ and $\psi<1$, Eq. (33) reduces to

$$
\frac{1}{\bar{r}^{2}} \frac{\partial}{\partial \bar{r}}\left(\bar{r}^{2} \frac{\partial \psi}{\partial \bar{r}}\right)=\underbrace{1+\psi}_{\text {ions }}-\underbrace{(1-\alpha)}_{\text {electrons }}
$$

or

$$
\frac{1}{\bar{r}^{2}} \frac{\partial}{\partial \bar{r}}\left(\bar{r}^{2} \frac{\partial \psi}{\partial \bar{r}}\right)=\psi+\alpha
$$

By considering $\psi+\alpha$ as the unknown, it is seen that $\psi+\alpha$ has solutions of the form $\bar{r}^{-1} \exp ( \pm \bar{r})$; the exponentially growing solution is allowed here because region 2 does not extend to infinity. A convenient way of expressing the general solution is

$$
\psi+\alpha=\frac{A \cosh \left(\bar{r}-\bar{r}_{o}\right)+B \sinh \left(\bar{r}-\bar{r}_{o}\right)}{\bar{r}} .
$$

In order to have $\psi=1 / \bar{Z}$ when $\bar{r}=\bar{r}_{o}$, we choose

$$
\frac{1}{\bar{Z}}+\alpha=\frac{A}{\bar{r}_{o}}
$$

and leave $B$ undetermined. Thus

$$
\psi_{2}=\frac{\bar{r}_{o}\left(\frac{1}{\bar{Z}}+\alpha\right) \cosh \left(\bar{r}-\bar{r}_{o}\right)+B \sinh \left(\bar{r}-\bar{r}_{o}\right)-\alpha \bar{r}}{\bar{r}}
$$

is the region 2 solution with coefficients arranged so that $\psi_{2}=1 / \bar{Z}$ when $\bar{r}=\bar{r}_{o}$.

\section{Region 1: Inner region}

In this region, $\psi>1$ and we assume that $\psi T_{i} / T_{e} \ll 1$ so that Eq. (33) reduces to

$$
\frac{1}{\bar{r}^{2}} \frac{\partial}{\partial \bar{r}}\left(\bar{r}^{2} \frac{\partial \psi}{\partial \bar{r}}\right)=\underbrace{\frac{2}{\sqrt{\pi}} \sqrt{\psi}}_{\text {ions }}-\underbrace{(1-\alpha)}_{\text {electrons }} \simeq \underbrace{\frac{2}{\sqrt{\pi}} \sqrt{\psi}}_{\text {ions }}
$$

where the electron term has been dropped because $1-\alpha$ is significantly less than unity and $\psi$ is assumed to be larger than unity. Equation (42) can be written as

$$
\frac{\partial^{2} \psi}{\partial \bar{r}^{2}}+\frac{2}{\bar{r}} \frac{\partial \psi}{\partial \bar{r}}=\mu(\psi) \psi,
$$

where

$$
\mu(\psi)=\frac{2}{\sqrt{\pi \psi}} \ll 1 .
$$

Since $\mu \ll 1$, the right-hand side of Eq. (43) may be neglected compared to either of the left-hand terms in which case the approximate solution to Eq. (43) is the vacuum-like solution

$$
\psi=\frac{c+d \bar{r}}{\bar{r}}
$$

where $c$ and $d$ are constants to be determined. The coefficient $d$ provides for the slight depression of the grain potential due to the shielding cloud. The $d$ term is allowed because region 1 is of finite extent and so finite $d$ is not inconsistent with $\psi$ vanishing at infinity since infinity is not located in region 1.

From Gauss' law, the radial electric field $E_{r}$ at the dust grain surface is

$$
4 \pi \epsilon_{0} r_{d}^{2} E_{r}=-Z_{d} e .
$$

Since $E_{r}=-\partial \phi / \partial r=\left(\kappa T_{i} / e \lambda_{\mathrm{D} i}\right) \partial \psi / \partial \bar{r}$, the boundary condition at the grain surface $r_{d}$ can be expressed as

$$
\left(\frac{\partial \psi}{\partial \bar{r}}\right)_{\bar{r}_{d}}=-\frac{Z_{d}}{4 \pi n_{i 0} \lambda_{\mathrm{Di}}^{3}} \frac{1}{\bar{r}_{d}^{2}} .
$$

This gives

$$
c=\frac{Z_{d}}{4 \pi n_{i 0} \lambda_{\mathrm{D} i}^{3}}
$$

and so, using Eqs. (7) and (12),

$$
c=\frac{\alpha \bar{a}^{3}}{3} \text {. }
$$


By assumption $\psi=1$ at $\bar{r}_{i}$ and so, using Eq. (45),

$$
\frac{c}{\bar{r}_{i}}+d=1
$$

in which case

$$
d=1-\frac{c}{\bar{r}_{i}} .
$$

Thus the region 1 potential is

$$
\psi_{1}=\frac{\frac{\alpha \bar{a}^{3}}{3}+\left(1-\frac{1}{\bar{r}_{i}} \frac{\alpha \bar{a}^{3}}{3}\right) \bar{r}}{\bar{r}}
$$

this satisfies Gauss's law at the dust grain surface and also gives $\psi=1$ at $\bar{r}=\bar{r}_{i}$. The potential on the grain surface is

$$
\psi_{d}=\frac{\alpha \bar{a}^{3}}{3}\left(\frac{1}{\bar{r}_{d}}-\frac{1}{\bar{r}_{i}}\right)+1
$$

\section{MATCHING THE SOLUTIONS}

\section{A. Matching principle}

Matching consists of arranging for equality of $\psi$ and $\psi^{\prime}$ at the two interfaces between the three collisionless regions; the necessity for continuity of $\psi$ and $\psi^{\prime}$ across an interface is established by integrating Eq. (33) twice across the interface. Solutions on the left- and right-hand sides of a matching radius $\bar{r}_{m}$ are of the general form $\psi_{\text {left }}=L(\bar{r}) / \bar{r}$ and $\psi_{\text {right }}$ $=R(\bar{r}) / \bar{r}$ and so matching requires

$$
\begin{aligned}
& L\left(\bar{r}_{m}\right) / \bar{r}_{m}=\psi_{m}=R\left(\bar{r}_{m}\right) / \bar{r}_{m}, \\
& L^{\prime}\left(\bar{r}_{m}\right)=R^{\prime}\left(\bar{r}_{m}\right),
\end{aligned}
$$

where $\psi_{m}=1 / \bar{Z}$ when $\bar{r}_{m}=\bar{r}_{o}$ and $\psi_{m}=1$ when $\bar{r}_{m}=\bar{r}_{i}$. Here $L$ and $R$ are the left- and right-hand numerators: $L$ is the numerator of $\psi_{1}$ and $R$ is the numerator of $\psi_{2}$ when $\bar{r}_{m}$ $=\bar{r}_{i} ; L$ is the numerator of $\psi_{2}$ and $R$ is the numerator of $\psi_{3}$ when $\bar{r}_{m}=\bar{r}_{o}$.

\section{B. Matching of $\psi_{2}$ and $\psi_{3}$ at $\bar{r}_{o}$}

The $\psi_{2}$ and $\psi_{3}$ solutions have already been arranged to satisfy Eq. (54) (i.e., $\psi_{2}=\psi_{3}=1 / \bar{Z}$ at $\bar{r}_{o}$ ). The derivative matching condition, Eq. (55), is satisfied if

$$
B=\alpha-\frac{r_{o}}{\bar{Z}} \sqrt{\alpha \bar{Z}+1}
$$

and so

$$
\psi_{2}=\frac{\bar{r}_{o}\left(\frac{1}{\bar{Z}}+\alpha\right) \cosh \left(\bar{r}-\bar{r}_{o}\right)+\left(\alpha-\frac{\bar{r}_{o}}{\bar{Z}} \sqrt{\alpha \bar{Z}+1}\right) \sinh \left(\bar{r}-\bar{r}_{o}\right)-\alpha \bar{r}}{\bar{r}}
$$

smoothly matches to $\psi_{3}$ at $\bar{r}_{o}$. The actual value of $\bar{r}_{o}$ is undetermined at this stage and will be found later.

\section{Matching of $\psi_{1}$ and $\psi_{2}$ at $\overline{\boldsymbol{r}}_{i}$}

Since Eq. (54) requires $R=\bar{r}_{i}$ in order to have $\psi\left(\bar{r}_{i}\right)$ $=1$, Eq. (57) provides the relation

$$
\begin{aligned}
\bar{r}_{i}= & \bar{r}_{o}\left(\frac{1}{\bar{Z}}+\alpha\right) \cosh \left(\bar{r}_{i}-\bar{r}_{o}\right) \\
& +\left(\alpha-\frac{\bar{r}_{o}}{\bar{Z}} \sqrt{\alpha \bar{Z}+1}\right) \sinh \left(\bar{r}_{i}-\bar{r}_{o}\right)-\alpha \bar{r}_{i} .
\end{aligned}
$$

The condition $L=\bar{r}_{i}$ when $\psi=1$ has already been arranged by the form of Eq. (52).

The condition $L^{\prime}=R^{\prime}$, found by taking derivatives of the numerators of Eqs. (52) and (57), is

$$
\begin{aligned}
1-\frac{\alpha \bar{a}^{3}}{3 \bar{r}_{i}}= & \bar{r}_{o}\left(\frac{1}{\bar{Z}}+\alpha\right) \sinh \left(\bar{r}_{i}-\bar{r}_{o}\right) \\
& +\left(\alpha-\frac{\bar{r}_{o}}{\bar{Z}} \sqrt{\alpha \bar{Z}+1}\right) \cosh \left(\bar{r}_{i}-\bar{r}_{o}\right)-\alpha .
\end{aligned}
$$

Equations (58) and (59) constitute two coupled equations in the unknowns $\bar{r}_{i}$ and $\bar{r}_{o}$. Using $\bar{Z} \gg 1$, these equations reduce to

$$
\begin{aligned}
& (1+\alpha) \bar{r}_{i}=\alpha \bar{r}_{o} \cosh \left(\bar{r}_{i}-\bar{r}_{o}\right)+\alpha \sinh \left(\bar{r}_{i}-\bar{r}_{o}\right), \\
& 1+\alpha-\frac{\alpha \bar{a}^{3}}{3 \bar{r}_{i}}=\alpha \bar{r}_{o} \sinh \left(\bar{r}_{i}-\bar{r}_{o}\right)+\alpha \cosh \left(\bar{r}_{i}-\bar{r}_{o}\right) .
\end{aligned}
$$

For given $\bar{a}$ and $\alpha$ these nonlinear equations can be solved numerically for $\bar{r}_{i}$ and $\bar{r}_{o}$. Since $\alpha=\alpha\left(\bar{a}, \bar{r}_{d}\right)$, this means that for any point in $\bar{a}, \bar{r}_{d}$ parameter space, one can calculate $\alpha$ and then calculate $\bar{r}_{i}$ and $\bar{r}_{o}$. Thus, we can consider $\bar{r}_{i}$ $=\bar{r}_{i}\left(\bar{a}, \bar{r}_{d}\right)$ and $\bar{r}_{o}=\bar{r}_{o}\left(\bar{a}, \bar{r}_{d}\right)$.

Once $\bar{r}_{i}$ and $\bar{r}_{o}$ are known, the solutions $\psi_{1}, \psi_{2}$, and $\psi_{3}$ are all determined and match smoothly across the interfaces. The Vlasov/Poisson equation is thus solved all the way from the grain surface to infinity. The potential falls off abruptly at $\bar{r}>\bar{r}_{o}$ with a scale length given by the dust Debye length. The dust Debye length is thus of physical importance even though it is much smaller than the inter-particle spacing. No paradoxes occur due to this situation because the solution for $\psi$ is multi-scale and more complicated than a simple Yukawa-type potential. In particular, the dust shielding does not take place in a sphere having a radius equal to the dust Debye length, but instead takes place over the surface of a 

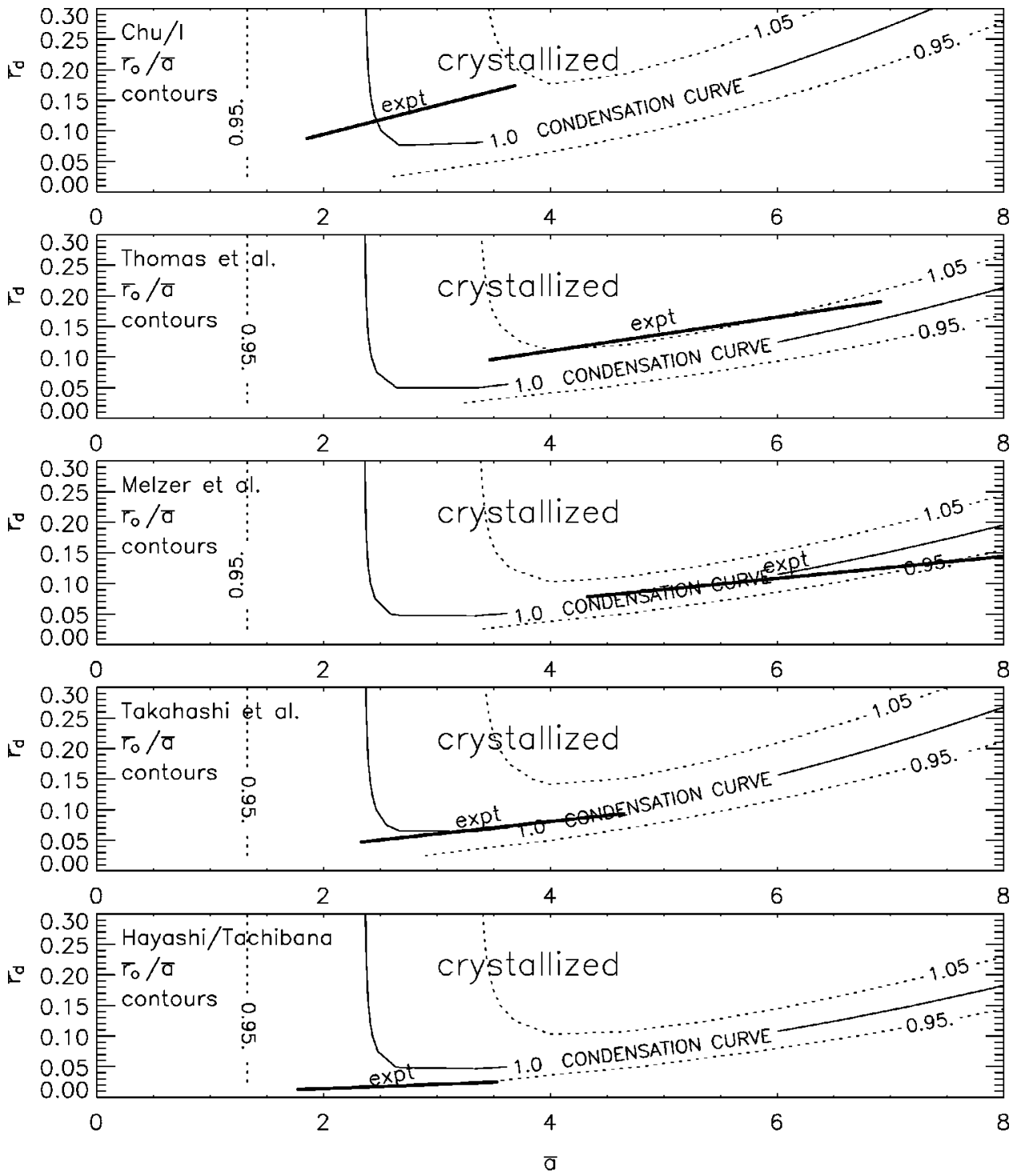

FIG. 3. Comparison of experiment parameters (short solid line marked "expt") with model prediction that dusty plasma will crystallize if experiment parameters intersect the condensation curve or are above it. sphere having a much larger radius (a few times the ion Debye length). The extremely sharp cut-off of $\psi$ beyond $\bar{r}_{o}$ completely decouples dust grains from each other if their interparticle separation distance exceeds $\bar{r}_{o}$.

\section{CRYSTALLIZATION}

When $\bar{a}>\bar{r}_{o}$ each dust grain is decoupled from neighboring dust grains and so the dust grains behave as a gas of non-interacting particles. However, if $\bar{a}<\bar{r}_{o}$, then each dust grain is within the shielding cloud of its neighbor and subject to the unshielded repulsive force of its neighbor. Because the repulsive force scales as $\bar{Z} \psi$, this repulsion becomes enormous as soon as a dust grain tries to move any significant distance inside of the $\bar{r}=\bar{r}_{o}$ layer (i.e., inside of the $\psi=1 / \bar{Z}$ layer). When experienced by other dust grains, a test particle dust grain thus acts like a hard sphere with radius $\bar{r}_{o}$. Thus, dust grains cannot move significantly inside $\bar{r}_{o}$ and so the condition for strong coupling and crystallization is that $\bar{a}$ becomes less than $\bar{r}_{o}$. The condensation curve is found by making the following sequence of calculations at each point $\bar{a}, \bar{r}_{d}$ in dusty plasma parameter space (i) calculate $\alpha\left(\bar{a}, \bar{r}_{d}\right)$, (ii) calculate $\bar{r}_{i}$ and $\bar{r}_{o}$ by solving the nonlinear coupled Eqs. (60) and (61), (iii) plot the locus of the curve $\bar{r}_{o}=\bar{a}$ and establish which side of this curve corresponds to $\bar{a}<\bar{r}_{o}$. The uppermost plot in Fig. 3 shows contours of constant $\bar{r}_{o} / \bar{a}$ calculated for Ref. 3 and marks the contour where $\bar{r}_{o} / \bar{a}=1$ as the "condensation curve;" above the condensation curve (and with $\bar{r}_{d} \ll 1$ as discussed earlier) the dusty plasma should be crystallized. It is seen that portions of the experiment line lie above the condensation curve, which means that the model predicts that the dusty plasma of Ref. 3 should be crystallized. Thus, there is excellent agreement between the model and the experimental parameters of Ref. 3 .

The other plots in Fig. 3 are similar, but use data from the experimental results reported by Thomas et al., ${ }^{4}$ Melzer et al., ${ }^{6}$ Hayashi and Tachibana, ${ }^{5}$ and Takahashi et al. ${ }^{28}$ There is excellent agreement between the model and all these experiments with the exception of the Hayashi/Tachibana experiment where the experimental curve lies slightly below the condensation curve. The upper part of Table I lists the parameters of these experiments while the lower part gives the results of dust charging theory and then the results of this 
TABLE I. Comparison between model predictions and experiments.

\begin{tabular}{|c|c|c|c|c|c|c|}
\hline First author and reference & & $\mathrm{Chu}^{3}$ & Thomas $^{4}$ & Melzer $^{6}$ & Takahashi $^{28}$ & Hayashi $^{5}$ \\
\hline Reported value & $n_{i 0}\left(\mathrm{~cm}^{-3}\right)$ & $10^{9}$ & $10^{9}$ & $2 \times 10^{8}$ & $10^{9}$ & $10^{9}$ \\
\hline Reported value & $n_{d 0}\left(\mathrm{~cm}^{-3}\right)$ & $2 \times 10^{5}$ & $4 \times 10^{4}$ & $1.4 \times 10^{3}$ & $10^{5}$ & $3 \times 10^{5}$ \\
\hline Reported value & $r_{d}(\mu \mathrm{m})$ & 5 & 5 & 12.5 & 5.4 & 1.3 \\
\hline Reported value & $T_{e}(\mathrm{eV})$ & 2 & 3 & 4 & 3 & 4.4 \\
\hline Reported value & $T_{i}(\mathrm{eV})$ & 0.03 & 0.025 & 0.03 & 0.03 & 0.025 \\
\hline From Eq. (1) & $a(\mu \mathrm{m})$ & 106 & 181 & 554 & 133 & 93 \\
\hline Neutral pressure & $\mathrm{Pa}$ & 16 & 200 & 80 & 87 & 0.3 \\
\hline Ion mean free path & $l_{\mathrm{mfp}}(\mu \mathrm{m})$ & $5 \times 10^{3}$ & $6 \times 10^{2}$ & $1.6 \times 10^{3}$ & $1.5 \times 10^{3}$ & $3 \times 10^{3}$ \\
\hline Ion mass & amu & 40 & 40 & 16 & 26 & 16 \\
\hline \multicolumn{7}{|l|}{ Modeled quantities } \\
\hline Ion density used in model & $n_{i 0}\left(\mathrm{~cm}^{-3}\right)$ & $10^{9}$ & $10^{9}$ & $10^{8}$ & $10^{9}$ & $10^{9}$ \\
\hline \multirow[t]{2}{*}{ From Eq. (5) } & $\lambda_{d i}(\mu \mathrm{m})$ & 41 & 37 & 128 & 41 & 37 \\
\hline & $4 \pi n_{i 0} \lambda_{\mathrm{D} i}^{3}$ & 842 & 641 & 2663 & 842 & 641 \\
\hline \multirow[t]{2}{*}{ From Eq. (7) } & $\bar{a}$ & 2.61 & 4.89 & 4.31 & 3.29 & 2.50 \\
\hline & $\bar{r}_{d}$ & 0.12 & 0.13 & 0.078 & 0.066 & 0.018 \\
\hline From Eq. (9) & $P$ & 0.021 & 0.0035 & 0.0029 & 0.0056 & 0.0034 \\
\hline From Eq. (10) & $\psi_{d}$ & 46 & 194 & 219 & 135 & 208 \\
\hline From Eq. (12) & $\alpha$ & 0.96 & 0.67 & 0.64 & 0.76 & 0.70 \\
\hline From Eq. (15) & $Z_{d}$ & $4.8 \times 10^{3}$ & $1.6 \times 10^{4}$ & $4.5 \times 10^{4}$ & $7.6 \times 10^{3}$ & $2.3 \times 10^{3}$ \\
\hline \multirow[t]{2}{*}{$\Gamma$} & $Z_{d}^{2} e^{2}$ & $10^{4}$ & $8.9 \times 10^{4}$ & $1.8 \times 10^{5}$ & $2.1 \times 10^{4}$ & $3.4 \times 10^{3}$ \\
\hline & $\overline{4 \pi \epsilon_{0} a \kappa T_{i}}$ & & & & & \\
\hline Solution of Eqs. (60), (61) & $\bar{r}_{i}$ & 1.51 & 3.76 & 2.99 & 2.03 & 1.20 \\
\hline \multirow[t]{2}{*}{ Solution of Eqs. (60), (61) } & $\bar{r}_{o}$ & 2.63 & 5.18 & 4.41 & 3.31 & 2.42 \\
\hline & $\bar{r}_{o} / \bar{a}$ & 1.005 & 1.06 & 1.02 & 1.005 & 0.97 \\
\hline
\end{tabular}

model using a best-fit density that is within experimental error. The main result is the values of $\bar{r}_{i}$ and $\bar{r}_{o}$. The $\bar{r}_{i}$ values in Table I greatly exceed $\bar{r}_{d}$ showing that the depression of the grain surface potential due to shielding is only a slight effect. Table I shows that when the published parameters of the experiments are used, a value of $\bar{r}_{o}$ is calculated which is slightly larger than $\bar{a}$; the calculated ratio $\bar{r}_{o} / \bar{a}$ is given in the bottom line of Table I. The fact that $\bar{r}_{o} / \bar{a}$ is greater than unity indicates that the experiment is above the condensation curve and so should be crystallized-this is our main result (the slight disagreement of the Hayashi/ Tachibana experiment will be discussed later). For reference, Table I also lists the value of $\Gamma$ associated with these experiments, and it is interesting to note that according to our model $\Gamma$ has no physical significance regarding condensation and so it is not surprising that $\Gamma$ has a range of quite different values for the different experiments.

It has not been possible to compare the model to the experiment under way ${ }^{8}$ on board the International Space Station, because plasma densities and temperatures have not yet been provided for that experiment.

Figure 4 shows plots of $\log \psi, \psi, \psi$ on an expanded scale (to show the behavior when $\psi \sim 1 / \bar{Z}$ ) $, n_{e} / n_{e 0}, n_{i} / n_{i 0}$, and $n_{d} / n_{d 0}$ for the Chu and I experiment ${ }^{3}$ using the values of $\bar{r}_{i}$ and $\bar{r}_{o}$ listed in Table I. The $\psi(\bar{r})$ plotted in Fig. 4 is calculated using Eq. (52) in region 1, Eq. (57) in region 2, and Eq. (35) in region 3; the electron, ion, and dust densities in Fig. 4 are calculated using Eqs. (20), (25), and (21), respectively. The dust temperature $T_{d}$ has been assumed to equal $T_{i}$ so that $\bar{Z}=Z_{d}$; different values of $T_{d}$ would only change the decay rate of $\psi$ outside of $\bar{r}_{o}$, but would not change the values of $\bar{r}_{i}$ and $\bar{r}_{o}$ since these are insensitive to the value of
$\bar{Z}$ as long as it is large compared to unity. It is seen that there is a sharp cut-off of the potential at $\bar{r}_{o}$ and that, beyond this radius, the potential decays precipitously with a characteristic scale length given by the dust Debye length. The potential curve is smooth all the way from the dust grain surface to infinity; this smoothness results from choosing $\bar{r}_{i}$ and $\bar{r}_{o}$ to match $\psi$ and its derivatives at the interfaces between collisionless regions.

A question arises regarding why the Hayashi/Tachibana experiment ${ }^{5}$ lies slightly below the condensation curve (i.e., has $\bar{r}_{o} / \bar{a}=0.97$ rather than above unity). Examination of the parametric sensitivity of the model predictions shows that intersection of the Hayashi/Tachibana experiment with the predicted condensation curve occurs if the assumed electron temperature is increased to $T_{e}=8 \mathrm{eV}$ or if the assumed grain diameter is doubled. Increasing the assumed atomic mass number to values larger than 16 also causes the experiment curve to approach the condensation curve but this effect is minimal in the relevant parameter range. Reference 5 reported an ion temperature which was not measured, but assumed, and an electron temperature which was estimated based on earlier measurements ${ }^{29}$ made in another plasma under similar conditions. It is possible therefore that the slight discrepancy between the model predictions and the Hayashi/ Tachibana experiment results from an inaccurate estimation of the electron to ion temperature ratio in the region of the dust grains. Better agreement would be obtained with a higher $T_{e} / T_{i}$ ratio and the values of $T_{e}$ and $T_{i}$ used in Table I were chosen to correspond to room temperature ions and the electron temperature measurement given in Fig. 4 of Ref. 29. Comparison with a scanning electron microscope mea- 


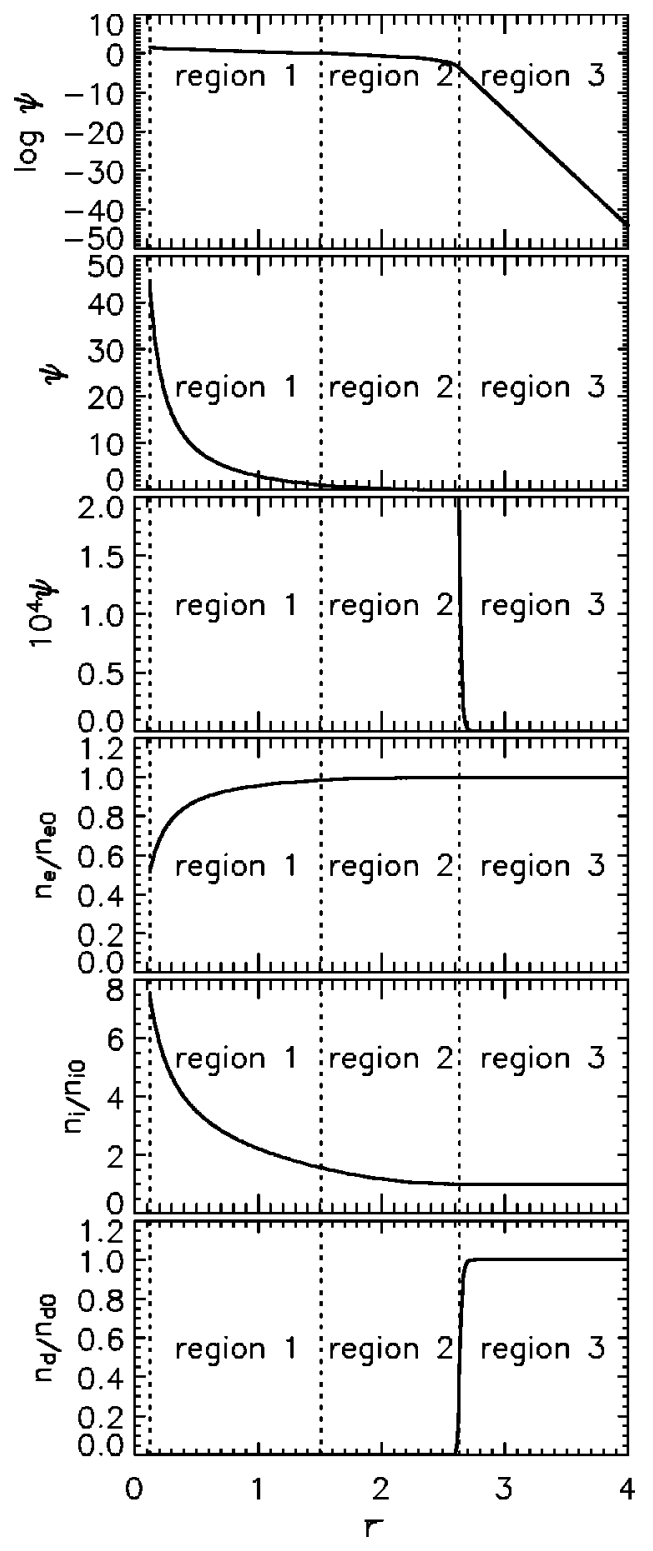

FIG. 4. Solutions for nominal parameters of Chu and I experiment as a function of $\bar{r}$. As shown in Table I, the relevant parameters are $T_{e}=2 \mathrm{eV}$, $T_{i}=0.03 \mathrm{eV}, \bar{a}=2.61, \bar{r}_{d}=0.12, \bar{r}_{i}=1.51, \bar{r}_{o}=2.63, \alpha=0.96$, and $\bar{Z}=4.8$ $\times 10^{3}$. From top to bottom plots are: $\log _{10} \psi, \psi, 10^{4} \psi$ which gives an expanded scale to show the region 3 decay, $n_{e} / n_{e 0}, n_{i} / n_{i 0}$, and $n_{d} / n_{d 0}$. The functional form of $\psi$ is determined from the appropriate asymptotic form in each of the three regions. Note the sharp cut-off of the dust density at $\bar{r}=\bar{r}_{o}$; the scale length of this cutoff is the dust Debye length $\lambda_{\mathrm{D} d}$.

surement has shown that the Mie scattering technique used by Hayashi/Tachibana to measure the dust grain diameter is quite accurate, ${ }^{30}$ so it is unlikely that the discrepancy between the model predictions and the Hayashi/Tachibana experiment is due to a factor of two error in measurement of the dust grain diameter.

Another question to be addressed is the possible importance of (i) barriers due to local maxima in the effective potential $^{12,26}$ and (ii) ion trapping/detrapping due to collisions. ${ }^{14-16}$ The model presented here argues that the amount of net charge in region 1 is so small that the potential in region 1 is nearly the same as the vacuum potential that would be produced by a bare, unshielded dust grain [see Eq.
(45)]. Changing the amount of charge in region 1 by factors as large as order unity would not affect this argument. Thus any reduction in the amount of charge in region 1 because of effective potential barriers will make no difference to the region 1 solution, because it is already assumed that there is no charge in region 1 . So long as the number of trapped ions in region 1 is small compared to $Z_{d}$, the potential in region 1 is mainly due to the dust charge and again it is reasonable to use the vacuum potential in region 1 . As for region 2, the ion density predicted by collisionless theory in region 2 is identical to the linearized Boltzmann relation obtained from collisional theory [see Eq. (37)]. Since trapping and detrapping result from collisions, trapping and detrapping should tend to make the system more Boltzmann-like, but since the system is already Boltzmann-like in region 2, trapping and detrapping should not cause significant changes to the region 2 ion density profile and thus should not significantly affect the $\psi$ profile in region 2. Effective potential barriers in region 2 may rearrange the radial charge distribution in region 2 slightly, but this should cause only a small effect on $\psi$ because $\psi$ is a double integral with respect to radius of the net charge distribution [see Eq. (32)]. As for region 3, the normalized potential $\psi$ is so small in region 3 that ions are unaffected by any spatial dependence of the potential; any corrections to the region 3 potential profile should therefore have negligible effect on ion trajectories. Thus, while effective potential barriers and trapping/detrapping may modify the net charge radial profile somewhat, these should have a much reduced effect on the $\psi$ profile and so should not cause any substantial changes in the values of $\bar{r}_{i}$ or $\bar{r}_{o}$. Small changes in the $\psi$ profile should not affect the basic premise that there exist three concentric collisionless regions each with distinct physics or the conclusion that dust grains condense when the radius $\bar{r}_{o}$ of the interface between regions 2 and 3 exceeds $\bar{a}$, the nominal intergrain spacing distance.

At this point in the discussion it is possible to revisit the assumption made at the end of Sec. III that ion capture by dust grains may be ignored when characterizing the collisionless ion velocity distribution function in regions $1-3$. Ignoring ion capture by the dust grain is tantamount to saying that all ions entering the collisionless region are reflected radially so that there are equal numbers of ions moving radially inward and outwards; if some ions were captured by the dust grain, there would be fewer ions moving radially outwards than inwards. The number of captured ions can be estimated using OML theory, ${ }^{31}$ which shows that the effective cross section for ions entering from a radius where the potential is zero and then being captured by a dust grain is $\sigma_{\text {capture }} \sim\left(1+\psi_{d}\right) \pi \bar{r}_{d}^{2}$. This capture cross section is to be compared to $\sigma_{\text {enter }}=\pi \bar{r}_{o}^{2}$, the cross section for ions to enter region 2 from outside (the outer boundary of region 2 is used because this denotes the edge of the potential well seen by the ions). Of the ions that enter region 2 , the fraction that are captured by the dust grain is given by the ratio $\sigma_{\text {capture }} / \sigma_{\text {enter }} \simeq\left(1+\psi_{d}\right) \bar{r}_{d}^{2} / \bar{r}_{o}^{2}$. Evaluation of this ratio using the Chu and I parameters in Table I $\left(\psi_{d}=46, \bar{r}_{d}=0.12, \bar{r}_{o}\right.$ $=2.63)$ gives $\sigma_{\text {capture }} / \sigma_{\text {enter }} \simeq 0.1$, which shows that the fraction of ions captured is small enough to be neglected (similar results hold for the other experiments listed in Table I). This 
validates the assumption made in Sec. III that distinctions ${ }^{20,26}$ between the outward and inward ion velocity distributions $f_{+}, f_{-}$may be neglected and confirms that Eq. (17) is a suitable representation for the ion distribution function.

\section{SUMMARY}

The standard linear fluid analysis of Debye shielding fails when $|q \phi / \kappa T|$ exceeds unity because the linear Debye shielding model is based on the assumption that $|q \phi / \kappa T|$ is small compared to unity. This issue is important for condensation of dusty plasmas, because condensation requires having $\left|Z_{d} e \phi / \kappa T_{d}\right|$ exceed unity.

Dusty plasmas can be characterized by an $\bar{a}, \bar{r}_{d}$ parameter space where $\bar{a}$ and $\bar{r}_{d}$ are the inter-grain spacing distance and grain radius normalized to the ion Debye length. An experiment corresponds to a point in this parameter space and if the density of the experiment is not known precisely, then the range of densities within experimental error corresponds to a slanted line segment in this parameter space.

Because shielding distances are much smaller than an ion collision mean free path, ions can be considered as collisionless in the shielding sphere surrounding a dust grain. A collisionless Vlasov model is used to calculate particle densities in the electrostatic potential of a dust grain test charge. This collisionless theory gives the same results as does Boltzmann theory for electrons and for dust grains because they are negatively charged but gives results different from Boltzmann theory for ions in the vicinity of the dust grain. Ions near a highly charged dust grain test particle fall into a deep potential well and are accelerated to high velocities. This means that no ions have zero velocity near the dust grain test particle and so integrals over the velocity distribution have a lower velocity limit corresponding to the minimum velocity of an ion falling into the deep potential well. This invalidates the fluid theory concept of pressure because pressure is based on the assumption of the existence of random velocities about some mean. For $\left|e \phi / \kappa T_{i}\right| \ll 1$, the ion density corresponds to the Boltzmann prediction, but for $\left|e \phi / \kappa T_{i}\right| \gg 1$ the ion density is much less than that predicted by the Boltzmann relation.

The potential in the vicinity of a dust grain test particle has three distinct types of behavior. These behaviors occur in concentric spherical regions consisting of (1) an inner region where the potential is vacuum-like, (2) a middle region where the potential includes both growing and decaying Yukawa-like terms with characteristic scale lengths of the order of the ion Debye length, and (3) an outer region with a rapidly decaying Yukawa-type solution having a scale length of the order of the dust Debye length. Region 1 physics differs from fluid theory, is consistent with dust charging physics, and avoids the paradoxes intrinsic to fluid theory at large $|e \phi / \kappa T|$.

For any point $\bar{a}, \bar{r}_{d}$ in dusty plasma parameter space, the requirement for smooth matching of the solutions at the interfaces between the three inner regions determines the locations $\bar{r}_{i}$ and $\bar{r}_{o}$ of these interfaces. Condensation occurs when $\bar{a}<\bar{r}_{o}$ and occurs on the line $\bar{a}=\bar{r}_{o}\left(\bar{a}, \bar{r}_{d}\right)$ which gives a "condensation curve" in dusty plasma parameter space; $\bar{a}$ is less than $\bar{r}_{o}$ above this curve and in this region the dusty plasma is crystallized. The model predicts condensation parameters in good agreement with published experiments.

${ }^{1}$ P. K. Shukla and A. A. Mamun, Introduction to Dusty Plasma Physics (IOP, Bristol, 2002).

${ }^{2}$ H. Ikezi, Phys. Fluids 29, 1764 (1986).

${ }^{3}$ J. H. Chu and L. I, Phys. Rev. Lett. 72, 4009 (1994).

${ }^{4}$ H. Thomas, G. E. Morfill, V. Demmel, J. Goree, B. Feuerbacher, and D. Mohlmann, Phys. Rev. Lett. 73, 652 (1994).

${ }^{5}$ H. Hayashi and K. Tachibana, Jpn. J. Appl. Phys., Part 1 33, 804 (1994).

${ }^{6}$ A. Melzer, T. Trottenberg, and A. Piel, Phys. Lett. A 191, 301 (1994).

${ }^{7}$ G. E. Morfill, B. M. Annaratone, P. Bryant, A. V. Ivlev, H. M. Thomas, M. Zuzic, and V. E. Fortov, Plasma Phys. Controlled Fusion 44, B263 (2002).

${ }^{8}$ A. P. Nefedov, G. E. Morfill, V. E. Fortov, H. M. Thomas, H. Rothermel, T. Hagl, A. V. Ivlev, M. Zuzic, B. A. Klumov, A. M. Lipaev, V. I. Molotkov, O. F. Petrov, Y. P. Gidzenko, S. K. Krikalev, W. Shepherd, A. I. Ivanov, M. Roth, H. Binnenbruck, J. A. Goree, and Y. P. Semenov, New J. Phys. 5, 33 (2003).

${ }^{9}$ W. L. Slattery, G. D. Doolen, and H. E. Dewitt, Phys. Rev. E 21, 2087 (1980).

${ }^{10}$ E. Wigner, Trans. Faraday Soc. 34, 768 (1938)

${ }^{11}$ D. R. Nicholson, Introduction to Plasma Theory (Krieger, Malabar, FL, 1992), Sec. 9.2.

${ }^{12}$ M. Lampe, G. Joyce, G. Ganguli, and V. Gavrishchaka, Phys. Plasmas 7, 3851 (2000).

${ }^{13}$ C. Hansen and J. Fajans, Phys. Rev. Lett. 74, 4209 (1995).

${ }^{14}$ J. Goree, Phys. Rev. Lett. 69, 277 (1992).

${ }^{15}$ A. V. Zobnin, A. P. Nefedov, V. A. Sinel'shchikov, and V. E. Fortov, JETP 91, 483 (2000).

${ }^{16}$ M. Lampe, R. Goswami, Z. Sternovsky, S. Robertson, V. Gavrishchaka, G. Ganguli, and G. Joyce, Phys. Plasmas 10, 1500 (2003).

${ }^{17}$ X. Wang and A. Bhattacharjee, Phys. Plasmas 3, 1189 (1996).

${ }^{18}$ N. Otani and A. Bhattacharjee, Phys. Rev. Lett. 78, 1468 (1997).

${ }^{19}$ H. M. Mott-Smith and I. Langmuir, Phys. Rev. 28, 727 (1926).

${ }^{20}$ J. E. Allen, R. L. F. Boyd, and P. Reynolds, Proc. Phys. Soc. London 70, 297 (1956).

${ }^{21}$ P. K. Shukla and A. A. Mamun, in Ref. 1, Eq. (2.2.1), p. 38.

${ }^{22}$ P. K. Shukla and A. A. Mamun, in Ref. 1, Eq. (2.3.4), p. 51.

${ }^{23}$ K. M. Watson, Phys. Rev. 102, 12 (1956).

${ }^{24}$ D. R. Nicholson, in Ref. 11, p. 72.

${ }^{25}$ J. G. Laframboise and L. W. Parker, Phys. Fluids 16, 629 (1973).

${ }^{26}$ I. B. Bernstein and I. N. Rabinowitz, Phys. Fluids 2, 112 (1959).

${ }^{27}$ M. Lampe, J. Plasma Phys. 65, 171 (2001).

${ }^{28}$ K. Takahashi, T. Oishi, K. Shimomai, Y. Hayashi, and S. Nishino, Phys. Rev. E 58, 7805 (1998).

${ }^{29}$ K. Tachibana, M. Nishida, H. Harima, and Y. Urano, J. Phys. D: Appl. Phys. 17, 1727 (1984).

${ }^{30}$ K. Tachibana and Y. Hayashi, Aust. J. Phys. 48, 469 (1995).

${ }^{31}$ P. K. Shukla and A. A. Mamun, in Ref. 1, Eqs. (2.3.2) and (2.2.6), pp. 38, 39. 